\title{
Healthcare workers teaching pilots: A satirical guide to breaking bad news
}

\section{By Peter Brindley and Martin Beed}

C ommercial aviation has long been held up as the exemplar of safety and reliability. Accordingly, healthcare organizations and clinicians have enthusiastically absorbed lessons from the airline industry: whether in simulation, human factors, or crisis management. Nurses and doctors have undoubtedly benefitted greatly from the ideas of pilots and flight crews. If so, then we owe it to our less earth-bound friends to share whatever applicable lessons we have in return. We offer the following reflection on breaking bad news from our high-stakes healthcare industry back to their high-stakes aviation industry, though with tongue planted firmly in cheek.

Satire is the use of humour, irony, exaggeration, or ridicule to make a more serious point. Our goal is to caution against the appealing but specious idea that the reality of one profession can be easily translated to another. Ultimately, while healthcare should always be eager to learn from others, perhaps the best analogy for healthcare is healthcare. If so then the best comparator for a poorly functioning hospital might also be a high-functioning hospital not an airline. Similarly, the best healthcare insights might be gained from looking at especially patient-focused wards, and the best hospitals in which to work may be those with high staff retention. In other words, while we should always look to other industries we should also celebrate and admonish the successes and failures in our own.

Those of us engaged in education and patient safety could also use an analogy that is closer-to-home, namely medical research. Trialists create studies where the intervention group and control group are closely matched. This is because they want conclusions that are generalizable, attributable, and free of bias. If educators and administrators do not use robust analogies then we create room for excuses and inaction. Comparing aviation and medicine is a useful conversation starter but comes with insoluble differences. For example, planes do not take off during inclement weather, or until the crew is familiar. Healthcare workers do not always have the luxury of time or choice when a patient is dying in front of them.

We shall focus on a skill that is increasingly important in emergency departments, namely how to break bad news. What follows is the invitation of an apocryphal airline Captain to attend our simulation centre for a four-part course in crisis communication. Even if we accept that many verbal evaluations are not worth the paper they are written on, we offer the following potential transcript:

\section{Session One: Structured debrief}

INSTRUCTOR: So, how do you feel that went?

CAPTAIN: Rather well, I thought. I quickly got one of the nurses take the family in to the relative's room. I then clearly explained that the patient was going to die.
INST: Yes, about that. It's certainly a good idea to take the family to a quiet room before breaking bad news, and it is prudent to take a nurse along. However, we don't usually ask the nurses to double check that the door to the room is locked.

\section{CAPT: Really? How strange!}

INST: Yes, and moreover, we traditionally go into the room with the relatives, rather than talking to them through the door.

CAPT: Really...you mean you talk to the relatives face-to-face? INST: That's correct.

CAPT: Only those from first class though. Surely you wouldn't... INST: We don't really separate our patients by class. I suppose you could say that we consider all of our patients to be "first class".

CAPT: Really. Goodness. Well I never.

***Transcript ends ${ }^{* * *}$

\section{Session Two: Structured debrief}

CAPTAIN: Well, I for one thought that went much better.

INST: Yes, you certainly took on the suggestions from the last session.

CAPT: Indeed, I did.

INST: I have a few comments, if you're uhm ready for take-off. CAPT: Absolutely, "chocks away" as we say.

INST: Well, if you remember, we discussed the need to give the relatives some sort of gentle opening statement. A few words that allow them to mentally prepare.

CAPT: Yes, indeed.

INST: Well I'm not sure I would go with "Brace! Brace! Brace!” I recommend going with something a little less alarming. Maybe a more soothing "I'm sorry, but I've got some bad news."

CAPT: Anything else?

INST: Well, now you mention it, having a checklist of points balanced on your knee doesn't go over well. Did you notice how they all looked a bit shocked when you ticked off the "is going to die" section?

CAPT: Well actually I don't look up when doing the checklist. INST: And I should point out that we don't normally offer the relatives a cup of coffee until after the conversation is over. It doesn't typically help having a resuscitation team member walk in halfway with a fully loaded drinks trolley, even if it does contain, as you pointed out, complementary wine and beer plus modestly priced sandwiches for those with a valid credit card.

*** Transcript ends ${ }^{* * *}$

\section{Session Three: Structured debrief}

CAPT: I must say, this breaking bad news malarkey is harder than it looks.

INST: Well, you did much better that time around.

CAPT: Well, the alterations to the scenario helped. I'm not really very good at all of the one-on-one stuff. It became much easier when I could pretend to talk to 250 passengers, all of who were 
going to die within minutes.

INST: Yes, and how did you find the idea of providing time for reflection?

CAPT: I felt the fact that I then said nothing more for ten minutes gave them more than enough time to contemplate the true meaning of death.

INST: Anything else?

CAPT: Well, I felt I demonstrated empathy and compassion. After all, I was also going to die.

***Transcript ends***

\section{Session Four: Structured debrief}

INST: Well, we thought we'd end on a tough scenario. Would you agree?

CAPT: Yes, I never considered that one might need the passengers' agreement before initiating a flight plan.

INST: Oh yes, autonomy is very important in modern healthcare decision-making.

CAPT: But, do the passengers know anything about planes or flying... I mean nursing and medicine?

INST: I'm sorry but I'll have to stop you there. That sort of "pilot knows best" has no place in modern communication skills training.

CAPT: But there are so many passengers ... I mean patients.

INST: Well, perhaps you could have asked the "passengers" to elect a spokesperson.

CAPT: But that's not what is expected in aviation law.

INST: Well yes, you see, you actually don't just need the passenger's agreement. I would also suggest you should have contacted their families.

CAPT: But it has nothing to do with them, they're not in the plane.

INST: Yes, but imagine the scenario - a passenger becomes unconscious during a flight. How would you know where they wanted to fly to? Who better than their family back home to tell you exactly where the plane needs to go?
CAPT: Uhm, yes, this communication is complicated stuff isn't it. I'd much rather just fly the plane.

${ }^{* * *}$ Transcript ends***

Perhaps in future sessions we could invite a group of clinicians to handwrite flight-plans for major airlines and then see exactly where the planes end up. We might then involve judges and lawyers in routine healthcare decision-making: what is the worst that could happen?

\section{About the authors}

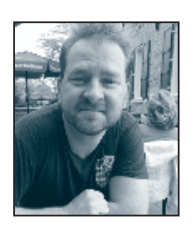

Dr. Peter Brindely, MD, FRCPC, FRCP (Lond), FRCP (Edin)

Peter is a full-time Critical Care Doc at the University of Alberta Hospital; Professor of Critical Care Medicine, Anesthesiology, and Medical Ethics. He has published over 100 peer-reviewed manuscripts, 25 book chapters, 50 lesser manuscripts on critical care in the prehospital, emergency department and intensive care settings. He has delivered over 400 invited presentations in 12 countries, and over 40 plenaries. He is convinced that happiness is about finding meaning and showing gratitude - he occasionally succeeds.

\section{Dr. Martin Beed, BMBS, FRCA, FFICM, DM}

Martin is a specialist in intensive care medicine and anesthesia, working in Nottingham University Hospital (UK). He has worked in various centres as far afield as Shetland, the most northerly islands of the British, and Royal Adelaide Hospital in Australia, where, from time to time, he was seconded to the Royal Flying Doctors Service. He is currently the chair of his hospital's resuscitation committee, has edited and contributed to several books on intensive care and anesthesia, and is a peer reviewer and writer for several high-profile medical journals. 